\title{
ON CALCULATION OF PARAMETERS OF ELECTRIC CIRCUITS OF SHIP PRESSURE-SEAL FEED-THROUGHS
}

\author{
Michael V. Teplyakov ${ }^{1}$, Margarita D. Loshenko ${ }^{2}$ \\ 1,2 "Scientific-Production Center" of \\ "Ship's Electrical Power System" LLC \\ 196128, Blagodatnaya Street 6, \\ Saint Petersburg, RUSSIA
}

\begin{abstract}
Relevance of the research lies in the fact that description of cable glands parameters such as electrical components of the line items, such as those characterized by the cables of any purpose are not represented in the literature and datasheets. Therefore, this article focuses on the calculations of the parameters of electric circuits. After all, the lack of consideration of these parameters can significantly affect the operation of complex electrical lines, consisting of several cables, their component cable glands and other connecting elements of electrical circuits. These studies confirmed that the characteristics of dielectric materials used in cable glands are changed when exposed to elevated temperatures and ambient pressure on of the electric line element parameters can be considered as a homogeneous cable line.
\end{abstract}

AMS Subject Classification: 49K45, 76J20, 93A30

Key Words: electric circuits, ship pressure-seal feed-throughs, parameters, cable line, PSFTs

\section{Introduction}

A ship pressure-seal feed-through (PSFT) is a complicated construction with

$\begin{array}{lr}\text { Received: } & \text { April 4, } 2017 \\ \text { Revised: } & \text { April 24, } 2017 \\ \text { Published: } & \text { May 8, } 2017 \\ \text { Correspondence } & \text { author }\end{array}$

(C) 2017 Academic Publications, Ltd. url: www.acadpubl.eu

\footnotetext{
$\S$ Correspondence author
} 
important functions in the course of operation (along with personnel life support). These are provision of strength balance of body design, tightness of releasable connection over the main body-PSFT body boundary, passage of any electrical circuits through the main body and continuity of shielding circuits.

The cable inlet may have up to three PSFTs. One of ship PSFTs is shown in Figure 1.

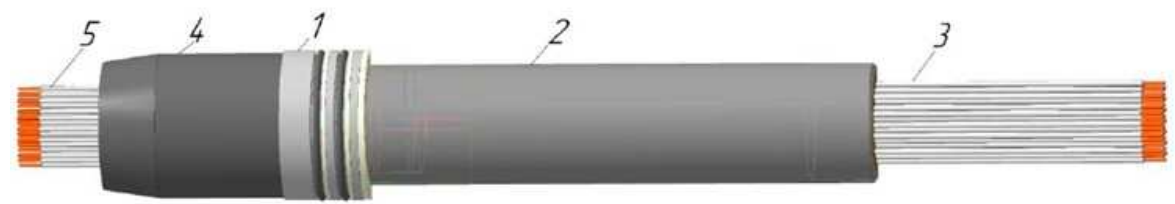

Figure 1: A ship PSFT: 1 - body of a pressure-seal insert; 2 - bushing; 3 wires; 4 -sealing element; 5 -current-carrying cable conductors.

A mathematical description of PSFT and properties that ensure its strength are given in [1], while a mathematical model describing behavior of PSFT as a sealing element is presented in [2]. A rather comprehensive consideration of PSFT constructions with respect to physics of processes occurring at PSFT exploitation is made in [3], [4] and [5]. However, the electrotechnical characteristics of PSFTs are presented in the existing literature mainly as results of experimental works and general features that are required to account for calculation of cable lines as well as electrodynamic and electrothermal stability.

In the technical literature, description of mutual influence between electrical circuits is presented insufficiently. It is limited by an analysis of the existing requirements of International Electrotechnical Commission (IEC) concerning the signaling lines for " 5 " and " $5+$ " categories [6]. In addition, the results of mathematical simulation of typical PSFTs are considered in [7]. For specified constructions and requirements, they confirm presence in parallel PSFTs lines of induced voltages comparable to running signals. However, there are no descriptions of parameters of PSFTs as components of current lines (similarly to those of any cables - capacitance, inductance, ohmic resistance of circuits) in both the literature and technical manuals.

\section{Materials and Methods}

Absence of allowance for these parameters can essentially affect operation of complex electrical circuits made of several cables with PSFTs and some other elements joining electrical circuits. Determination of PSFT circuit parameters is 
complicated by the facts that conductors off the sealed inserts have the structure and geometry not similar to those of cables, are parallel to each other, have bends and other non-system departures.

Let us consider a sequence of elements of the PSFT electrical circuits to determine its possible departure of ohmic resistance from that of the calibration sample. We take a wire segment of length equal to the total PSFT length as the calibration sample. The wire ohmic resistance as well as isolation parameters are fixed by the standard technical documentation. Figure 2 presents the elements of PSFT electrical circuit.
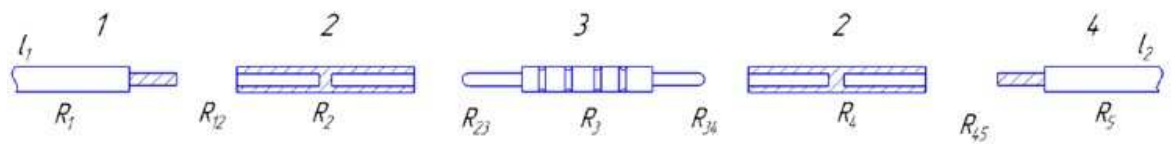

Figure 2: The elements of PSFT electrical circuit: 1 - current-carrying cable conductor; 2 - connection element; 3 - contact bar; 4 - wire.

When several elements are joined together into a line using the known methods (soldering, flattening, screw joint, joining with a spring split element, etc.), some additional elements (with the corresponding ohmic resistance) are connected into an electrical circuit. In this case, a diagram for calculation of PSFT ohmic resistance is such as shown in Figure 3:

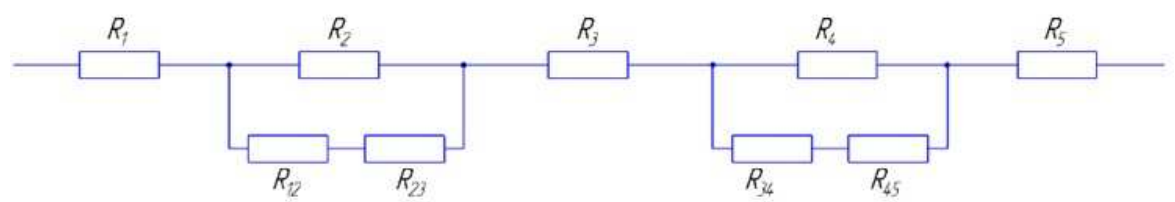

Figure 3: A diagram for calculation of PSFT ohmic resistance.

Then the expression for calculating ohmic resistance of any electrical circuit of PSFT will be

$$
R=R_{1}+\frac{R_{2}\left(R_{12}+R_{22}\right)}{R_{2}+R_{12}+R_{23}}+R_{3}+\frac{R_{4}\left(R_{34}+R_{45}\right)}{R_{4}+R_{34}+R_{45}}+R_{5}
$$

Another critical parameter that must be ensured for PSFT exploitation is insulation resistance $R_{i}$ of circuits with respect to the body as well as to each other. This parameter is ensured when making PSFT and has to be maintained in the course of PSFT exploitation. Based on the elements presented in Figure 2 , the equivalent circuit for calculating $R_{i}$ of PSFT circuits is that shown in Figure 4. 


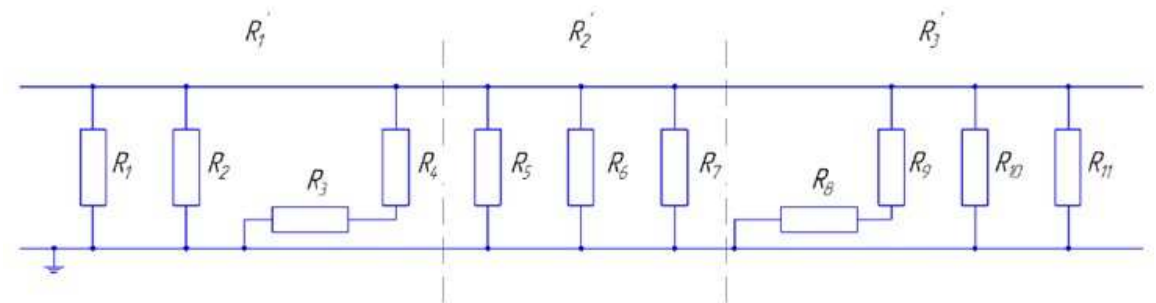

Figure 4: The equivalent circuit for calculating $R_{i}$ of PSFT circuits: $R_{1}$ is $R_{i}$ of the standard insulating material of wire; $R_{2}$ is $R_{i}$ of the standard insulating material of wire, heat shrink tube and sealant compound; $R_{3}$ is $R_{i}$ of the heat shrink tube; $R_{4}$ is $R_{i}$ of the sealant compound to the point nearest the body;

$R_{5}$ is $R_{i}$ of the sealant compound in the region of glass (or some other insulating material formed in the course of making) joining the point nearest the body; $R_{6}$ is $R_{i}$ of glass or other insulator to the point nearest the body; $R_{7}$ is $R_{i}$ similarly to $R_{4}$ on the opposite side; $R_{8}\left(R_{9}\right)$ is analog of $R_{3}\left(R_{4}\right)$; $R_{10}\left(R_{11}\right)$ is analog of $R_{2}\left(R_{1}\right) ; R_{1^{\prime}}, R_{2^{\prime}}$ and $R_{3^{\prime}}$ are ohmic resistances of the first, second and third parts of the equivalent circuit, respectively.

Let us present the equivalent circuit as a sum of three parts whose ohmic resistances are as follows:

$$
\begin{gathered}
R_{1}^{\prime}=\frac{R_{1} R_{2}\left(R_{3}+R_{4}\right)}{R_{1} R_{2}+R_{2}\left(R_{3}+R_{4}\right)+R_{1}\left(R_{3}+R_{4}\right)} \\
R_{2}^{\prime}=\frac{R_{5} R_{6} R_{7}}{R_{5} R_{6}+R_{6} R_{7}+R_{5} R_{7}} \\
R_{3}^{\prime}=\frac{\left(R_{8}+R_{9}\right) R_{10} R_{11}}{\left(R_{8}+R_{9}\right) R_{10}+\left(R_{8}+R_{9}\right) R_{11}+R_{10} R_{11}}
\end{gathered}
$$

Then the design equation for $R_{i}$ of PSFT circuits may be presented as:

$$
R_{i}=\frac{R_{1}^{\prime} R_{2}^{\prime} R_{3}^{\prime}}{R_{1}^{\prime} R_{2}^{\prime}+R_{2}^{\prime} R_{3}^{\prime}+R_{1}^{\prime} R_{3}^{\prime}}
$$

\section{Results}

The above circuits and equations describe the characteristics of PSFT circuits that obey the Ohm's law. The sufficiently important cable characteristics are inductive reactance of cable conductors and capacitance per unit length; they have essential effect on the line parameters at elevated frequencies. 
The length of a ready-fitted PSFT is over $2.0 \mathrm{~m}$. The arrangement of conductors in the major part of PSFT is irregular, i.e., the wires are chaotically situated along the PSFT axis. Presence of dissimilar dielectrics along the length of electrical circuits leads to physical non-uniformities in the structure of cable lines with PSFTs; they have to be taken into account. The electrical signals passing through PSFT are very manifold. Some data on their characteristics are presented in Table 1.

Table 1. Characteristics of electrical signals passing through PSFT.

\begin{tabular}{lllll}
\hline SI & Voltage, V & Current, A & $\begin{array}{l}\text { Signal fre- } \\
\text { quency, } \mathrm{Hz}\end{array}$ & Signal form \\
\hline 1 & 380 & 6 & 50 & sinusoidal \\
\hline 2 & 5 & 0.16 & 5000 & pulsed \\
\hline 3 & +450 & 0.01 & no data & pulsed \\
\hline 4 & -450 & 0.01 & no data & pulsed \\
\hline 5 & +100 & 0.01 & no data & pulsed \\
\hline 6 & -100 & 0.01 & no data & pulsed \\
\hline 7 & 5 & 0.05 & 875 & pulsed \\
\hline 8 & no data & no data & $1.25 \times 10^{8}$ & digital \\
\hline
\end{tabular}

A uniform cable line is characterized by the primary electrical parameters (active circuit resistance $R$, inductance $L$, capacitance $C$ and conductance $G$ ) per unit length of line. (Conductance $G$ is determined by $R_{i}$ of separate electrical circuits relative to the body and between each other.)

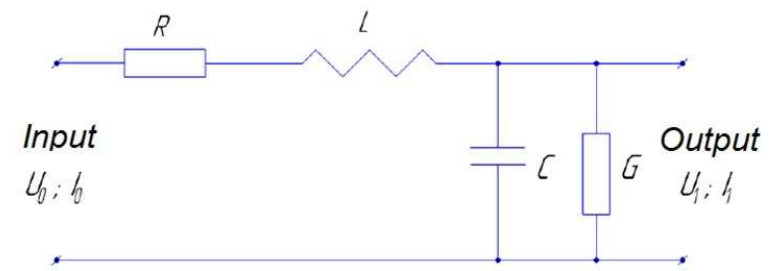

Figure 5: The equivalent circuit of a cable line: $R, L, C$ and $G$ are active resistance, inductance, capacitance and conductance of conductors, respectively.

By their physical nature, the primary electrical parameters of a cable are similar to those of oscillator circuits. However, contrary to the latter, they are not lumped but are distributed over the whole length of cable line. Thus, a cable line with a PSFT is that with distributed parameters. Its parameters in 
the PSFT section will be different from those of cables directly connected to PSFT.

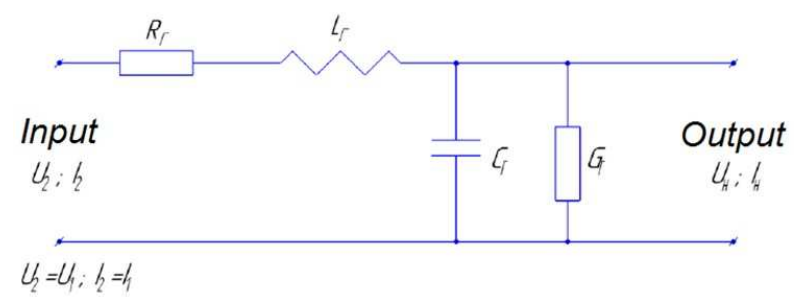

Figure 6: The equivalent circuit of a cable line in PSFT section; $R_{P S F T}, L_{P S F T}, C_{P S F T}$ and $G_{P S F T}$ are active resistance, inductance, capacitance and conductance of PSFT, respectively.

So the equations of a cable line with a PSFT are:

$$
\begin{aligned}
& \frac{-d \dot{U}}{d x}=\dot{I}\left(R_{P S F T}+j \omega L_{P S F T}\right) \\
& \frac{-d \dot{I}}{d x}=\dot{U}\left(G_{P S F T}+j \omega C_{P S F T}\right)
\end{aligned}
$$

Their solutions for voltage and current at an arbitrary point $\mathrm{x}$ of the cable line are:

$$
\begin{gathered}
\dot{U}_{x}=\dot{U}_{0} \operatorname{ch} \gamma x-\dot{I}_{0} \dot{Z}_{w} \operatorname{sh} \gamma x \\
\dot{I}_{x}=\dot{I}_{0} \operatorname{ch} \gamma x-\frac{\dot{U}_{0}}{\dot{Z}_{w}} \operatorname{sh} \gamma x
\end{gathered}
$$

Here $Z_{w}$ is wave resistance of the cable line (PSFT) section, $\gamma$ is a signal propagation constant in the cable line.

We suppose that in our case the total energy is absorbed by the load, i.e., there is no signal reflected from the cable line end. This is because the cable lines with PSFTs use double-wire lines (or even multiple-wire lines) rather than coaxial ones. In this case, Eqs. (8) and (9) are as follows:

$$
\begin{gathered}
\dot{U}_{x}=\dot{U}_{0} \operatorname{ch} \gamma x \\
\dot{I}_{x}=\dot{I}_{0} \operatorname{ch} \gamma x
\end{gathered}
$$

Investigation of different dielectrics (Figure 7) (from among those used in PSFTs and whose properties differed from those of cable materials) that were in closed space showed that their properties changed under pressure. The samples studied were capacitor cells (Figure 8); they were investigated using an original test bench making it possible to vary temperature and pressure. The parameters were measured with an $L C R$ meter $G W$ Instek LCR-7816. 


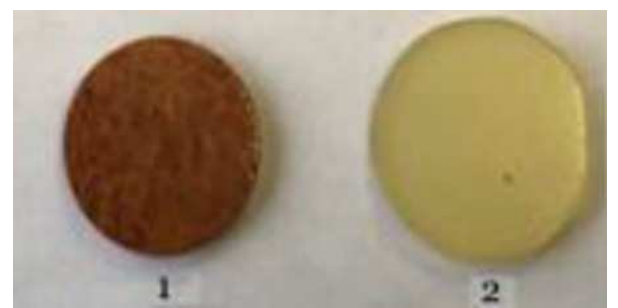

Figure 7: Examples of dielectric materials: 1 - DCV-21, 2 -K-115 compound.

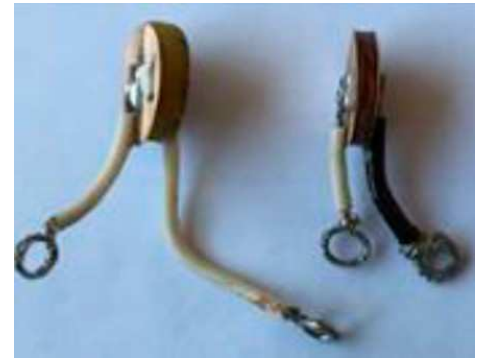

Figure 8: Capacitors made on the basis of examples of dielectric materials.

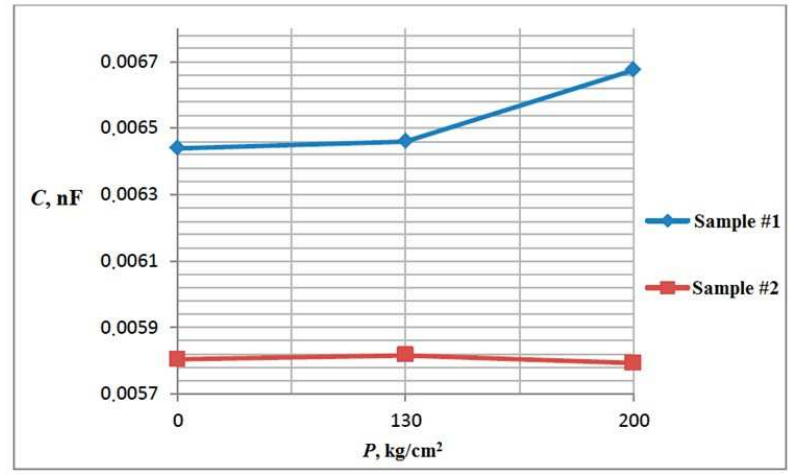

Figure 9: Effect of elevated pressure $P$ on properties of dielectrics.

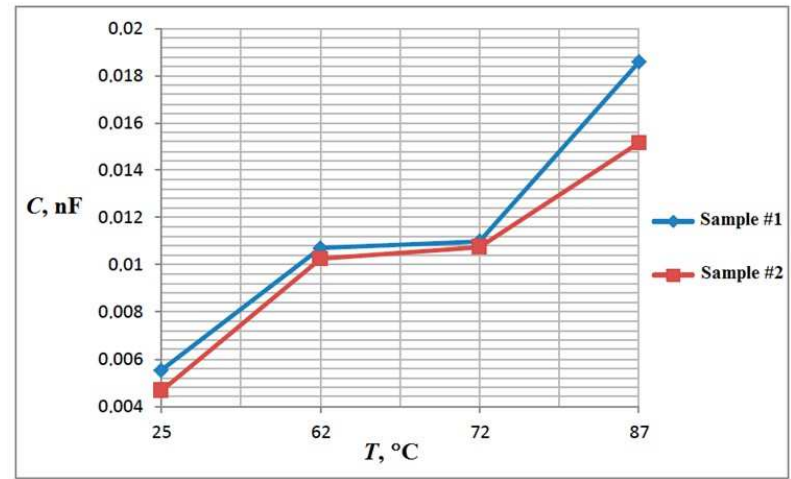


Figure 10: Effect of elevated temperature $T$ on properties of dielectrics.

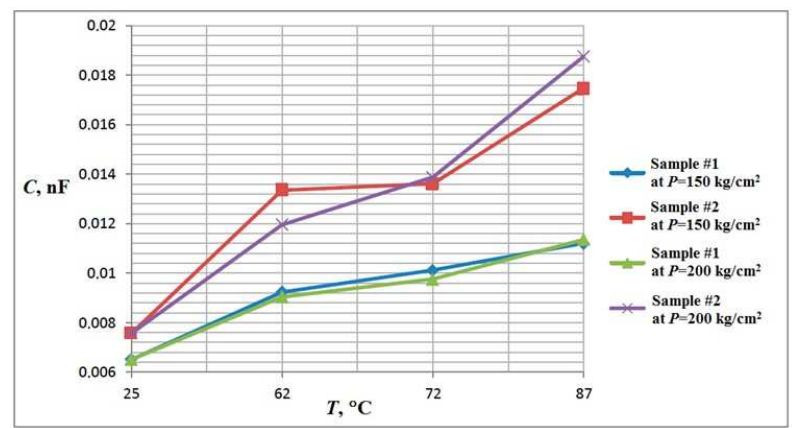

Figure 11: Effect of elevated pressure $P$ and temperature $T$ on properties of dielectrics.

For a cable line with PSFT, the expressions for voltage and current are as follows:

$$
\begin{gathered}
\dot{U}_{x}=\dot{U}_{0} \operatorname{ch} \gamma x+\dot{U}_{1} \operatorname{ch} \gamma x \\
\dot{I}_{x}=\dot{I}_{0} \operatorname{ch} \gamma x+\dot{I}_{1} \operatorname{ch} \gamma x
\end{gathered}
$$

The measurements were performed at an input signal frequency of $1000 \mathrm{~Hz}$. The results of investigations made over a wider frequency range are presented in [8] and [9]. One can conclude that characteristics of dielectrics depend on frequency, especially in the low-frequency (below $10 \mathrm{kHz}$ ) spectral range.

Based on the desired parameters of signals transmitted over a cable line with PSFTs and taking into account the effect of operational factors on dielectric properties, one can suppose that characteristics of cable lines with PSFTs vary during operation depending on ambient temperature and pressure, so the value of capacitance $C$ may vary over some range.

\section{Conclusions}

Being a part of a cable line, a ship PSFT may be considered as a uniform cable line when calculating parameters of electrical circuit elements.

2. A wire segment of length equal to the total PSFT length may be considered as a calibration sample when calculating PSFT ohmic resistance $R$ and insulation resistance $R_{i}$ of circuits with respect to the body as well as to each other. 
3. The results of the studies made confirm that characteristics of the dielectric materials used in PSFTs change under the action of elevated temperature and ambient pressure.

4. In the course of exploitation, the characteristics of cable lines with PSFTs vary depending on ambient temperature and pressure. Capacitance $C$ of a system of conductors can vary within certain limits; this affects the leakage currents, especially at frequencies up to $10 \mathrm{kHz}$.

\section{References}

[1] M.V. Teplyakov, M.D. Khazieva, On assumptions when simulation modeling and calculating strength of ship current feed-throughs, Sudostroenie, No. 3 (2014), 30-35.

[2] N.A. Lazarevskii, M.V. Teplyakov, On permeability mechanisms of current feed-throughs, Sudostroenie, No. 6, (2012) 40-41.

[3] M.V. Teplyakov, Ship Pressure-seal Feed-throughs for Extreme Operating Conditions. The Theory and Design Manual, TsNRS Publishing, Novosibirsk (2014).

[4] M.V. Teplyakov, A.V. Selivanov, M.D. Khazieva, On calculation of stability of ship current feed-throughs under fire of models, Privolzhskii Research Herald No. 5 (2014), 46.

[5] M. Teplyakov, M. Khazieva, K. Syupov, On the calculation of the interference levels of simulation models of ship current leads, in Scientific enquiry in the contemporary world: theoretical basics and innovative approach, B\&M Publishing, USA (2014).

[6] M.V. Teplyakov, On the effect of frequency converters for electrical drives on data lines, Morskoi Vestnik, Special issue No. 2 (2013), 77-80.

[7] M.V. Teplyakov, M.D. Khazieva, On a version of calculation of a ship current feedthrough using some mathematical model, Nauka i Sovremennost, No. 27 (2014), 178.

[8] N.Z. Strizhevskii, Coaxial Videolines, Radio i Svyaz Publishing, Moscow (1988).

[9] I.I. Grodnev, N.A. Frolov, Coaxial Communication Cables, Radio i Svyaz Publishing, Moscow (1983). 
